\title{
The Application and Research of Remote Video Surveillance System
}

\author{
Sun Xinxin \\ Department of Computer science and information \\ Zhejiang Water Conservancy And Hydropower College \\ Hangzhou, China \\ e-mail: sunxx@zjwchc.com
}

\begin{abstract}
This article is on the application and research of remote surveillance system in the statement of the functions of video surveillance system. It overall introduces a whole set of the system structure of video surveillance system, the general design of the hardware and the communication protocol in the process of application.
\end{abstract}

Keywords-video surveillance, audio, long-distance, system structure, communication protocol

\section{INTRODUCTION}

With the rapid development of network technology, image processing technology and video compression coding technology, the application of remote surveillance technology has been increasingly popular, which plays an important role in a number of fields. As a component of security and protection system, video surveillance system is able to observe the area under surveillance directly through controlled camera and other auxiliary equipment, like Pan Tilt Zoom (PTZ), lens etc. It can record all or part of the pictures and audios in the area under surveillance to serve as the important condition and evidence for future reference. Remote video surveillance is a value-added service providing pictures, audios and all kinds of alarming signals through remote collecting, transmitting, storing and broadcasting for customers in the use of broadband network. This service enables users to realize the real-time monitoring, surveillance supervision, video storage and alarm linkage with no limit of time and place. Therefore, it is with great significance of the application of remote video surveillance technology under the networking environment.

\section{SyStem STRUCTURE OF REMOTE VIDEO SURVEILLANCE}

Remote video surveillance system is computer soft and hardware system in the realization of remote live surveillance and control through network system. This article provides a model of remote surveillance system which monitors real life situation, and exchanges the real-time data and controlled information through internet and central server so that clients are able to use browser to visit web server through internet. Figure 1 illustrates the system structure model of remote surveillance system.

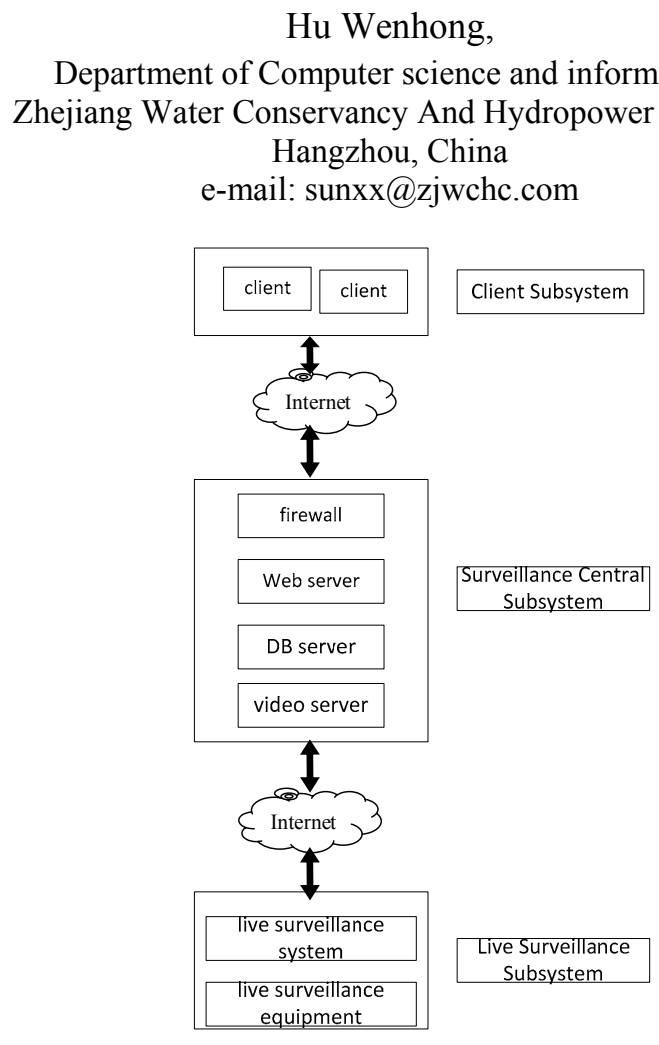

Figure 1. remote surveillance system consist

A. System Structure Model of Remote Surveillance System

From Figure 1, we can learn that the whole remote surveillance system consist three parts, namely live surveillance subsystem, surveillance central subsystem and client subsystem.

Live surveillance subsystem: including all audio and video collecting system, signal transmission path, signal receipt and transmission equipment and other surveillance and storage equipment etc. It is in charge of monitoring each live area under surveillance, transmitting to real-time surveillance equipment after collecting and pre-processing and passing data to surveillance central subsystem. On the other hand, it receives the controlling orders from surveillance central subsystem and analyses and verifies the orders and then takes relevant actions. When the system equipped with surveillance software, monitoring personnel could make live surveillance through that subsystem and control and supervise the operation of surveillance equipment. In the research of surveillance system according to this article, the forefront surveillance equipment uses DVR/DVS. The collection and compression of video in DVR are integrated. In this system, the camera analog video signal 
is transmitted to DVR card. The analog signal then is exchanged to digital signal through video collection chips and then to temp-storage of the card. After that, the compression calculation is conducted on the card video compression trip which compresses the huge video signal to smaller and finally the compressed data is stored on the hardware. In addition, the collection of audio is directly realized through Serial COM485 of DVR via collecting digital signal by using live noise meter.

Surveillance central subsystem: it is the core of the whole remote surveillance system, made of firewall, web server, database server, video proxy server etc. It is in charge of the management, coordination and maintenance of the whole networking surveillance system. Video server responds to the requests of clients, on the one hand, it accepts their requests and takes the real-time data from the database or forefront storage equipment and then publishes on the web browser for users to observe; on the other hand, it stores the controlled orders from clients to the database for live controlling center to collect.

Client subsystem: is the direct interactional part with users. It receives the transmission from the users, obtains remote surveillance data from or to surveillance central subsystem and conducts the remote surveillance and diagnosis of the equipment through surveillance center. Clients do not need to install any professional software but on the ordinary personal computer with Web browser. When distant clients need to make surveillance to one certain equipment, it, in the first place, logs on the central website through remote terminal browser. After user authentication, it finds the relevant controlled website of the equipment and downloads the surveillance controlled software. With the connection of controlled software and equipment, it realizes the surveillance to the displayed surveillance interface of the equipment. The realized path is through internet with the means of rented specialized circuit or public database websites. During the real processing, the web security must be guaranteed which can be achieved by the technologies including firewall, user authentication etc.

\section{B. Hardware Structure of Video Surveillance System}

In the process of remote surveillance system, the detailed hardware structure is displayed in Figure 2. According to some networking features, the only thing users need to do is to type in the name of camera or office adopted by $\mathrm{C} / \mathrm{S}$ structure or $\mathrm{B} / \mathrm{S}$ structure on this platform. All tasks should be completed at the back stage automatically.

The forefront camera, noise meter etc. are used to collect the live audio and video analog signals. Through the specialized circuit, the collected video analog signals are transmitted to DVR/DVS, and changed to digital format before compression and encoding. The shot video data is recorded as the format of media files and functions of playing and replaying analysis. DVR/DVS supports the transmission of audio and video UDP and TCP. Central server as the authentication center of the whole surveillance system is in charge of achieving each authentication of longdistance logging of the users and each surveillance operation. These functions can be basically realized through software.
Clients can browse the real-time images through web browser. In the whole system, audio data can be collected through audio collection software in DVR. It is the noise data through transparent channel of COM 485 by per-second real-time monitored noise meter. It buffers the noise data and sends the noise data on the collection software in line with transmission protocol located on surveillance central server so as to realize central storage. When clients make surveillance requests, the latest noise data will be displayed in the user browser as line graph.

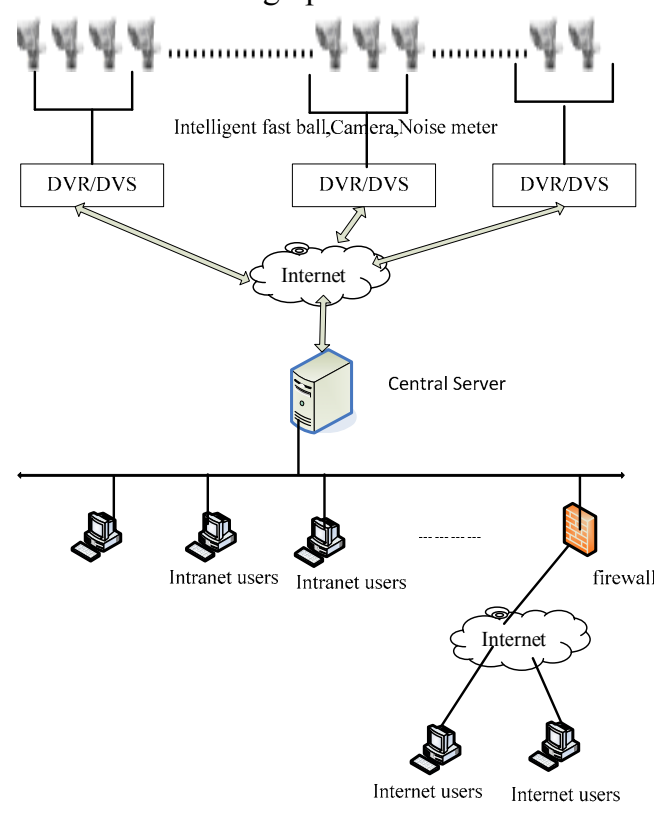

Figure 2. Hardware Structure

\section{The Structure of System Software}

In surveillance system, the software operated is video branch console management software, video networking browse software, security management software, system management software, video proxy server software, noise collection software, noise collection transmission software and Pan Tilt Zoom (PTZ) controlling signal transmission software.

The structure of software adopts mixing format of Three Platforms, Two Frames' . The so-called two frames are: Client/Server Networking Model, Browser/Server Model. That is to say, clients make the requests to server, which makes relevant responses and conducts its tasks, for example, clients send multicast address, image format and compression format etc. After building the connection, PC users are able to monitor each area under remote surveillance at clients' ends, which receive the video information from the remote monitored area. They can make orders, for example, image switching, camera rotation etc., to the servers under surveillance during the operation. The server makes controlling orders to the real-time equipment according to users' requests so as to realize the remote surveillance. The three platforms stand for security 
protection platform, software support platform, networking communication platform. Figure 3 demonstrates the structure of the whole system. Database adopts Postgres7.2, Oracle.

Application within the C/S Frame: main operation image information management software, video proxy server management software and security setting, system management software. Application within the B/S Frame: main operation networking inquiry orientation and networking browse software.

The layout of each part in the whole system is depicted in Figure 4. In fact, during the real practice, the functions of command center, authentication server, DB server, video proxy server, Web server can be integrated on one central server.

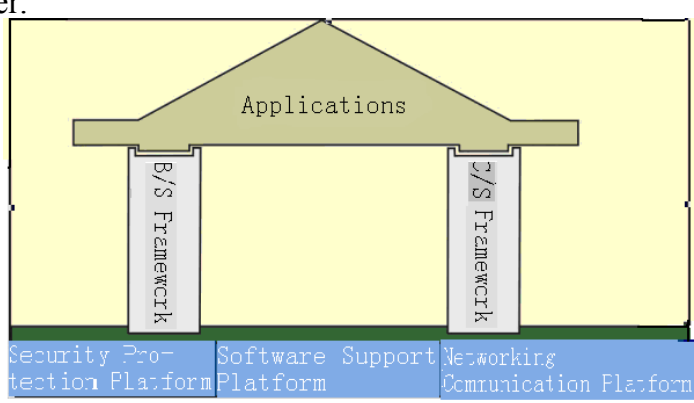

Figure 3. The Structure of System Software

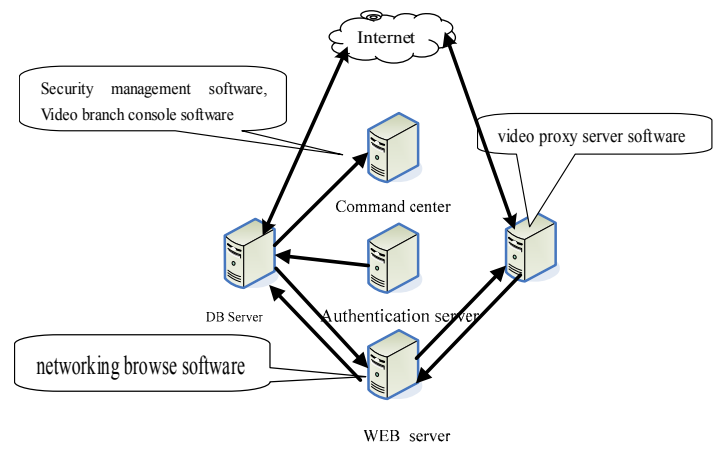

Figure 4. The Layout of Software

Video networking browse, as the webpage format, visits real-time images and recorded image data. It has the functions such as registration, logging, and authentication. According to access control and security strategy system will allocate relevant images to users to realize broadcasting and control as well as to the recorded broadcasting of image file including real-time noise check-up and noise research. After logging in, the partial functions of video branch console management software will be realized through $\mathrm{B} / \mathrm{S}$, therefore, users are able to visit, control and allocate the forefront DVS/DVR through IE directly. Clients do not need to install any $\mathrm{C} / \mathrm{S}$ application program because the software is operated on Web server. This browse software enables users directly visit through IE hence the security is of great significance. Because HTTP mechanism is the discontinuing connection between clients and servers, theoretically, any user on the internet is able to realize the direct visit, the security strategy must be reinforced. At the website, dynamic real-time transmission between DVR/DVS and clients is realized by ActiveX which in turn is as a small program. It can be operated in other application programs rather than IE. Theoretically, after users log off the video websites system, ActiveX still can be operated with prominent security issue. Therefore, users must log in when using the software so that system identifies their visiting authority according to their information. Only online users can have the relevant right to visit Active $\mathrm{X}$ as to ensure the security of the system. In order to guarantee the visiting security, it is of importance to have security communication protocol. In the whole system, the lower communication protocol still adopts UDP while higher one follows customized answering TCP/UDP protocol.

\section{System COMMUNICATION PROTOCOL}

UDP protocol is the transmission protocol facing nonconnection, which is by no means of ensuring mechanism of providing data transmission. Before transmitting data, no virtual connection needs to be made, neither adopts responding confirmation nor retransmission mechanism. Therefore, in the sound networking environment, the efficiency is better than TCP protocol. Taking such advantage of UDP and together with TCP/IP transmission control protocol, high-efficient transmission can be ensured as well as its reliability. Since UDP is not responsible for information reliable transmission mechanism, but to complete the functional transition, like security and ordering, etc. to higher level which is the application level, which greatly reduces operation time. Therefore, the real-time services such audio and video adopts UDP communication method to transmit data. However, if regarding to controlling information, TCP protocol will be adopted due to small data volume and high accuracy.

The types of security communication protocol are as follows:

(1) Request protocol format:

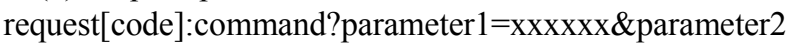

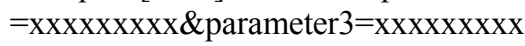

(2) Respond protocol format:

response[code]:command?parameter $1=x x x x x x \&$ parameter

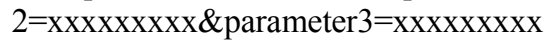

Notes: code is the visiting serials. If there exists code in request, the relevant response should also have code which should be the same as the one in the request for the use of judging and distinguishing from which request the response comes from. Command is the order for request and response. Parameter is the transmission specification, with the symbol $\&$ in between. The symbol of ? is for division between orders and parameters.

In the real audio and video signal transmission, both TCP protocol and UDP protocol exist and coordinate with each other. Specific customized protocol is not introduced here. 


\section{CONCLUSION}

The whole surveillance system integrates the surveillance equipment and noise meter to form a highly integrated whole-some equipment, which is able to realize the remote tracking management of live audio and video situations. System operation, so far, has successfully solved the problem of unavailability of relevant live surveillance regarding to departments concerned that achieves the unprecedented extensiveness of monitoring scope.

\section{REFERENCES}

[1] Chenlong, engineering of security and protection system. Beijing Tsing Hua University Publishing House, 2002

[2] Yanglei, lifeng,Tian Yansheng, Closed circuit television system ( 2nd Edition), Beijing China Machine Press, 2003

[3] Yang Zhixiong, Research on computer surveillance system mode of medium and small-size hydropower station

[4] Wangwenlue Taohongyan etc. Research on equipment remote surveillance technology, Computer engineering and application, 2005 